\title{
GROUPS WHICH HAVE A FAITHFUL REPRESENTATION OF DEGREE LESS THAN $p-1$
}

BY

WALTER FEIT(1)

1. Introduction. The object of this paper is to prove the following result which was conjectured by $\mathrm{R}$. Brauer in a conversation with the author.

THEOREM. Let $G$ be a group of order $g$ which has a faithful representation of degree n over the complex numbers. Let $g=g_{1} h$ where $\left(g_{1}, h\right)=1$ and $p>n+1$ for every prime $p$ dividing $h$. Then $G$ contains a normal abelian subgroup $K$ of order $h$ or $h / p$ for some prime $p\left({ }^{2}\right)$.

In a previous paper [5], the author and J. G. Thompson investigated groups which satisfy the more stringent condition $p>2 n+1$. The general outline of the proof of the theorem above is quite similar to the proof used in [5]. However, since there are two possibilities in the conclusion of the theorem, induction of necessity forces one to investigate several cases where only one occurred in the argument used in [5].

The theorem is of course vacuously true if $h$ is a prime. However, in the course of the proof it is often necessary to use detailed information concerning the irreducible characters of a group whose order is divisible by only the first power of a prime. The relevant information which is needed is contained in $\$ 3$ and is essentially due to R. Brauer $[2 ; 3]$ and H. F. Tuan [7]. At this point the theory of modular characters appears to be unavoidable. We also utilize the fact proved by $N$. Ito [6], that if a solvable group $G$ satisfies the hypotheses of the theorem, then $G$ contains a normal subgroup of order $h$.

2. Notation. All groups in this paper are assumed to be finite.

If $X$ is a group then $X^{\prime}$ and $Z(X)$ denote the commutator subgroup and center of $X$ respectively. If $Y$ is a subset of $X$ then $Y \triangleleft X$ means that $Y$ is a normal subgroup of $X$. If $A, B$ are subsets of $X$, then $A-B$ means set theoretic difference. $\cup$ means set theoretic union.

Received by the editors April 15, 1963.

(1) This work was partly supported by O.O.R. contract DA-30-115-ORD-976.

(2) Mr. D. Winter has informed me that in case the inequality is replaced by $p=2 n+1$, he has not only proved this theorem but has determined the structure of the groups in question. 
If $S$ is a subset of $X$ then:

$\langle S\rangle$ is the group generated by $S .\langle 1\rangle$ and 1 will generally be identified, $S^{*}=S-\langle 1\rangle$,

$|S|$ is the cardinality of $S$,

$C_{X}(S)$ is the centralizer of $S$ in $X$,

$N_{X}(S)$ is the normalizer of $S$ in $X$,

A subset $S$ of $X$ is a trivial intersection set in $X$ or a T.I. set in $X$ if

(i) $S \subseteq N_{X}(S)$,

(ii) $x^{-1} S x \cap S \subseteq\langle 1\rangle$ for all $x \in X-N_{X}(S)$.

For any complex valued class functions $\alpha, \beta$ on $X$ define

$$
(\alpha, \beta)_{X}=\frac{1}{|X|} \sum_{X} \alpha(x) \overline{\beta(x)}
$$

where - denotes complex conjugation, and $\|\alpha\|_{X}^{2}=(\alpha, \alpha)_{X}$. The subscript $X$ is omitted whenever it is clear from context which group is involved.

If $\alpha$ is a character of $X$ then the kernel of the representation with character $\alpha$ is also called the kernel of $\alpha$. Furthermore $\alpha$ is said to be faithful if its kernel is $\langle 1\rangle$. If $\beta$ is another character of $X$ then $\beta \subseteq \alpha$ means that $\alpha-\beta=0$ or $\alpha-\beta$ is a character of $X$. If $\alpha$ is a class function of a subgroup of $X$ then $\alpha^{*}$ denotes the class function of $X$ induced by $\alpha$.

$1_{X}$ is the principal character of $X$.

$\rho_{X}$ is the character of the regular representation of $X$.

If $p$ is a prime then a character $\alpha$ is $p$-rational if its field of values is linearly disjoint from the field of $p^{n}$ th roots of unity over the rationals. Two characters $\alpha, \beta$ are $p$-conjugate if there exists an automorphism $\sigma$ of the field generated by the values of $\alpha$ such that $\sigma(\alpha)=\beta$ and $\sigma$ leaves all $m$ th roots of unity in any extension field fixed for any $m$ such that $p \nmid m$.

For convenience we state here a result which is a generalization of Lemma 1 in [5]. The proof used there yields the following statement.

LEMMA 2.1. If $Y \triangleleft X$ and $x$ is an element of $X$ such that $C_{X}(x) \cap Y=\langle 1\rangle$, then $\lambda(x)=0$ for any irreducible character $\lambda$ of $X$ which does not have $Y$ in its kernel.

3. Groups which have a Sylow subgroup of prime order. Lemma 3.1 is proved by the methods of R. Brauer (see [2;3]), and is a simple consequence of his results. It was stated without proof by Tuan [7, Lemma 4]. Most of the proof could be recast so as to make no reference to the theory of modular characters. However the only known proof of the important relations (3.4) and (3.5) makes use of the modular theory. Only the statement of the lemma will be used in the sequel. 
Lemma 3.1. Let $p$ be a prime and let $X$ be a group whose Sylow p-group $P$ has order $p$. Let $e=\left[N_{X}(P): C_{X}(P)\right]$. Let $Y$ be the normal subgroup of $X$ generated by $P$. Assume $P$ is not normal in $X$ and $X$ has an irreducible character $\omega$ such that $\omega(1)<(p-1)$ and $\omega_{\mid Y}$ is a faithful character of $Y$. Then $Y=Y^{\prime}$ and there exists a linear character $\lambda$ of $C_{X}(P)$ which does not have $P$ in its kernel such that if $\tilde{\lambda}$ denotes the character of $N_{X}(P)$ induced by $\lambda$ then one of the following possibilities must occur

(i) $\omega_{\mid P}=\rho_{P}-\tilde{\lambda}_{\mid P}$ and $\omega(1)=p-e \geqq(p+1) / 2$.

(ii) $\omega_{\mid P}=\tilde{\lambda}_{\mid P}$ and $\omega(1)=e=(p-1) / 2$.

Proof. We will first show that it suffices to prove the lemma for groups $X$ satisfying $X=X^{\prime}=Y$. Suppose this has been done. Let $\omega_{\mid Y}=\sum_{i=1}^{s} \omega_{i}$, where each $\omega_{i}$ is an irreducible character of $Y$. Let $Y_{i}$ be the kernel of $\omega_{i}$. Since $Y \triangleleft X, \omega_{i}(1)$ $=\omega_{1}(1)$ for $1 \leqq i \leqq s$. Thus if $s>1$, then $\omega_{i}(1)<(p-1) / 2$ and hence $P Y_{i} \triangleleft Y$ [5, Theorem 1]. Since $\bigcap_{i} Y_{i}=1$, this yields that $P \triangleleft Y$ and so $P \triangleleft X$ contrary to assumption. Hence $\omega_{\left.\right|_{Y}}$ is irreducible.

If $Y \neq Y^{\prime}$, then there exists $Y_{1} \triangleleft Y$ such that $\left[Y: Y_{1}\right]=p$. Let $q$ be any prime dividing $\left|Y_{1}\right|$ and let $Q$ be a Sylow $q$-group of $Y_{1}$ which is normalized by $P$. Then $P Q$ is a solvable group and thus (see [6]) $Q \subseteq N_{Y}(P)$. Hence $\left|Y^{\prime}\right||| N_{Y}(P) \mid$ and so $P \triangleleft Y$ contrary to assumption. Hence $Y=Y^{\prime}$.

Since $\omega$ is a class function on $X$, the lemma applied to $Y$ yields that $\left[N_{Y}(P): C_{Y}(P)\right]=e$. Thus the result follows for $X$ once it is known for $Y$.

We will now assume that $X=X^{\prime}=Y$ and hence $\omega$ is a faithful character of $X$.

Let $C=C_{X}(P)$ and let $N=N_{X}(P)$. Then $N / C$ is a cyclic group of order $e$ and $e \mid(p-1)$. For any class function $\alpha$ of $C$ let $\alpha^{N}$ be the class function of $N$ induced by $\alpha$. A well-known theorem of Burnside $[8$, p. 173] implies that

$$
C=P \times V
$$

for some group $V$.

We have [3, Theorem 1 and Corollary 2$]$ that $e \leqq(p-1) / 2$ and either

$$
\omega_{\mid P}=\theta(1)\left\{\rho_{P}-\lambda_{\mid P}^{N}\right\}
$$

or

$$
\omega_{\mid C}=(\lambda \theta)_{\mid C}^{N},
$$

for some nonprincipal linear character $\lambda$ of $C / V$ and some irreducible character $\theta$ of $C / P$.

If (3.1) holds then

$$
(p-1)>\omega(1)=\theta(1)(p-e) \geqq \theta(1)\left(\frac{p-1}{2}\right) .
$$

Thus $\theta(1)<2$ and so $\theta(1)=1$. This shows that case (i) of the lemma occurs. 
Assume now that (3.2) holds. It suffices to show that $e \geqq(p-1) / 2$. Since in that case $e=(p-1) / 2$ and (3.2) yields that

$$
(p-1)>\omega(1)=e \theta(1)=\frac{p-1}{2} \theta(1) .
$$

Therefore $\theta(1)<2$ and so $\theta(1)=1$. Case (ii) of the lemma now follows from (3.2).

Let $B_{1}$ be the $p$-block of $X$ which contains $1_{X}$. Then $[2 ; 3]$

$$
B_{1}=\left\{\alpha_{i}\right\} \cup\left\{\beta_{j}\right\} \cup\left\{\chi_{k}\right\}
$$

where each $\alpha_{i}$ and $\beta_{j}$ is $p$-rational, $\left\{\chi_{k}\right\}$ is a family of $p$-conjugate characters and

$$
\left|\left\{\chi_{k}\right\}\right|=\frac{p-1}{e}, \quad\left|\left\{\alpha_{i}\right\} \cup\left\{\beta_{j}\right\}\right|=e .
$$

Furthermore for all $i$ and $j$

$$
\begin{aligned}
& \alpha_{i \mid P}=1_{P}+a_{i} \rho_{P}, \\
& \beta_{j \mid C}=-1_{C}+b_{j} \rho_{C / V}+\theta_{j} \rho_{C / V},
\end{aligned}
$$

for some characters $\theta_{j}$ of $C / P$ orthogonal to $1_{C}$ and integers $a_{i}, b_{j}$. If $\left\{\lambda_{k}^{N}\right\}$ is the set of all distinct characters of $N$ induced by nonprincipal linear characters $\lambda_{k}$ of $C / V$, then $\left|\left\{\lambda_{k}^{N}\right\}\right|=(p-1) / e$ and the notation can be chosen so that

$$
\chi_{k \mid P}=-\delta \lambda_{k \mid P}^{N}+c \rho_{P}, \quad \delta= \pm 1
$$

for all $k$ and some integer c. Equations (3.4), (3.5) and (3.6) yield that [2, Theorem 11]

$$
\delta \chi(1)+\sum_{i} \alpha_{i}(1)=\sum_{j} \beta_{j}(1)
$$

for $\chi=\chi_{k}$.

Since $P$ is not in the kernel of $\omega$ and $\omega(1)<p-1, \omega$ is not $p$-rational. Let $\omega^{\prime}$ be a character of $X$ distinct from $\omega$ which is $p$-conjugate to $\omega$. Then [3, Theorem 2]

$$
\begin{aligned}
& \omega^{\prime} \bar{\varrho} \supseteq \sum_{j} \beta_{j \mid c}, \\
& \omega^{\prime} \bar{\omega} \supseteq \chi_{\mid c} \text { if } \delta=-1
\end{aligned}
$$

where $\delta$ is defined in (3.6) and $\chi=\chi_{k}$ for a suitably chosen $k$. By (3.2)

$$
\left(\omega^{\prime} \bar{\omega}_{\mid P}, 1_{P}\right)_{P}=0 \text {. }
$$

Assume first that $\delta=-1$ in (3.6). Then (3.6), (3.9) and (3.10) imply that

$$
\chi_{\mid P}=\lambda_{\mid P}^{N},
$$

for some linear character $\lambda$ of $C / V$. Hence $\chi(1)=e$. Let $K$ be the kernel of $\chi$. Then 
$X / K$ has no normal subgroup of order $p$ since $X=X^{\prime}=Y$. Thus [3, Theorem 3] $e \geqq(p-1) / 2$ which suffices to complete the proof in this case. Hence it may be assumed that $\delta=1$. Therefore (3.6) implies that

$$
\chi(1) \geqq p-e .
$$

By (3.5), (3.8) and (3.10) we get that for all $j$

$$
\beta_{j \mid C}=\rho_{C / V}-1_{C}, \quad \beta_{j}(1)=p-1 .
$$

Since $X=X^{\prime},(3.4)$ implies that for all $i$

$$
\alpha_{i}(1) \geqq p+1 \text { if } \alpha_{i} \neq 1_{X} .
$$

Hence if $u=\left|\left\{\beta_{j}\right\}\right|,(3.3),(3.7)$ and (3.11) yield that

$$
u(p-1) \geqq 1+(p-e)+(e-u-1)(p+1) .
$$

Therefore

$$
2 u \geqq e, \quad u=\left|\left\{\beta_{j}\right\}\right| .
$$

For any character $\zeta$ of $C$, let $\zeta^{\prime}$ be the p-conjugate character of $\zeta$ under the automorphism which sends $\omega$ into $\omega^{\prime}$. Let $x_{1}, \cdots, x_{e}$ be coset representation of $C$ in $N$ and for any character $\zeta$ of $C$ define $\zeta_{i}$ by

$$
\zeta_{i}(y)=\zeta\left(x_{i}^{-1} y x_{i}\right) \text { for } y \in C .
$$

Then by (3.2)

$$
\omega_{\mid C}=\sum_{i=0}^{e-1} \lambda_{i} \theta_{i}
$$

and so

$$
\omega^{\prime} \bar{\omega}_{\mid C}=\sum_{i=0}^{e-1} \sum_{j=0}^{e-1} \lambda_{i}^{\prime} \bar{\lambda}_{j} \theta_{i} \bar{\theta}_{j}
$$

Since $\left(\theta_{i} \bar{\theta}_{j}, 1_{c}\right)=0$ or 1 according to whether $\theta_{i} \neq \theta_{j}$ or not, (3.14) implies that

$$
\omega^{\prime} \bar{\omega}_{\mid C}=\sum_{i, j}^{\prime} \lambda_{i}^{\prime} \bar{\lambda}_{j}+\zeta
$$

where $\zeta$ is a sum of irreducible characters of $C$, none of which have $V$ in their kernel, while the summation ranges over all pairs $(i, j)$ with $\theta_{i}=\theta_{j}$. Hence (3.8) and (3.12) imply that

Thus by (3.12)

$$
\sum_{i, j}^{\prime} \lambda_{i}^{\prime} \lambda_{j} \supseteq \sum_{j} \beta_{j \mid c}
$$

$$
u(p-1)=\sum_{j} \beta_{j}(1) \leqq \sum_{i, j}^{\prime} \lambda_{i}^{\prime} \lambda_{j}(1) \leqq e^{2}
$$


Consequently (3.13) implies that $e((p-1) / 2) \leqq e^{2}$, or $(p-1) / 2 \leqq e$. As was seen earlier this is sufficient to complete the proof of the lemma in all cases.

4. Some preliminary results. The lemmas proved in this section are generalizations of corresponding results in [5] and are proved by the methods of [5]. For convenience the basic assumption is stated separately.

HyPOTHESIS 4.1. (i) $Y$ is an abelian subgroup of the group $X, N=N_{X}(Y)$, $Z=Z(X),[N: Y Z]=t$ and

$$
N_{0}=\left\{\bigcup_{y \in Y^{*}} C_{X}(y)\right\}-Z .
$$

(ii) $Y$ and $N_{0}$ are T.I. sets in $X$ and $N_{0} \subseteq N_{X}\left(N_{0}\right)=N$.

(iii) $N \neq X$.

It follows immediately from Hypothesis 4.1 that $Y \cap Z=1$. Thus

$$
Y^{*} \subseteq N_{0} \subseteq N=N_{X}\left(N_{0}\right) \text {. }
$$

Lemma 4.1. Assume that Hypothesis 4.1 is satisfied. Let $\chi$ be an irreducible character of $X$ which does not have $Y$ in its kernel. Then

$$
\left(\chi_{\mid Y}, 1_{Y}\right)_{Y}<\frac{1}{|Y|^{1 / 2}} \chi(1) .
$$

Proof. Let

$$
\chi_{\mid N}=\alpha+\beta,
$$

where $\beta$ has $Y$ in its kernel and no irreducible constituent of $\alpha$ has $Y$ in its kernel. Let $b=\beta(1)$. Then $b=\left(\chi_{\mid Y}, 1_{Y}\right)_{Y}$. Furthermore $|\beta(x)|=b$ for $x \in Y Z$. Hence Lemma 2.1 and Hypothesis 4.1 imply that

$$
\begin{aligned}
1 & =\|\chi\|^{2}>\frac{1}{|X|} \frac{|X|}{|N|} \sum_{N_{0}}|\chi(x)|^{2} \\
& \geqq \frac{1}{|N|}\left\{-\sum_{Z}|\chi(x)|^{2}+\sum_{N_{0} \cup Z}|\alpha(x)+\beta(x)|^{2}\right\} \\
& \geqq \frac{-\chi(1)^{2}|Z|}{|N|}+\|\alpha\|_{N}^{2}+(\alpha, \beta)_{N}+\overline{(\alpha, \beta)_{N}}+\frac{1}{|N|} \sum_{Y Z}|\beta(x)|^{2} \\
& =-\frac{\chi(1)^{2}}{t|Y|}+\|\alpha\|_{N}^{2}+\frac{b^{2}}{t} .
\end{aligned}
$$

As $Y$ is not in the kernel of $\chi$ we get that $\alpha \neq 0$ and so $\|\alpha\|_{N}^{2} \geqq 1$. Hence

$$
\frac{\chi(1)^{2}}{t|Y|}>\frac{b^{2}}{t}
$$

which is equivalent to the statement to be proved. 
Lemma 4.2. Assume that Hypothesis 4.1 is satisfied. Assume further thet $Y$ is not contained in any proper normal subgroup of $X$. Let $\chi$ be a nonlinear irreducible character of $X$. Then

$$
\chi(1)+1>|Y|^{1 / 2} \text {. }
$$

Proof. Let $\chi_{0}=1_{X}, \chi_{1}=\chi, \chi_{2}, \cdots$ be all the irreducible characters of $X$. Define

$$
\begin{aligned}
& a_{i}=\left(\chi_{i}, \chi \bar{\chi}\right), \\
& b_{i}=\left(\chi_{i \mid Y}, 1_{Y}\right),
\end{aligned}
$$

for all $i$. The Frobenius reciprocity theorem implies that

$$
b_{i}=\left(\chi_{i}, 1_{Y}^{*}\right) \text {. }
$$

Therefore

$$
\begin{aligned}
\sum_{i} a_{i} b_{i} & =\left(\chi \bar{\chi}, 1_{Y}^{*}\right) \\
& =\frac{\chi(1)^{2}}{|Y|}+\frac{1}{|N|} \sum_{Y^{*}}|Z| t|\chi(y)|^{2} \\
& =\frac{1}{|Y|} \sum_{Y}|\chi(y)|^{2}=\left\|\chi_{\mid Y}\right\|^{2} .
\end{aligned}
$$

Since $Y$ is abelian, $\chi(1) \leqq\left\|\chi_{\mid Y}\right\|_{Y}^{2}$. As $a_{0}=b_{0}=1$, Lemma 4.1 now yields that

$$
\chi(1)-1 \leqq \sum_{i \neq 0} a_{i} b_{i}<\frac{1}{|Y|^{1 / 2}} \sum_{i \neq 0} a_{i} \chi_{i}(1)=\frac{\chi(1)^{2}-1}{|Y|^{1 / 2}} .
$$

As $\chi$ is nonlinear this implies that $|Y|^{1 / 2}<\chi(1)+1$ as required.

5. The proof of the main theorem: preliminary reductions. Throughout the remainder of this paper let $G$ be a counter example of minimum order to the theorem stated in $\S 1$. Let $\chi$ be the character of a faithful representation of $G$ of minimum degree. We will derive a contradiction from the assumed existence of $G$.

(5A) $G$ contains an abelian subgroup $H$ of order $h$. Furthermore $h$ is not a prime.

Proof. The existence of $H$ is known [4, p. 349]. Since $G$ is a counterexample to the main theorem $h$ is not a prime.

(5B) $H \cap Z(G)=1$.

Proof. Let $H_{1}=H \cap Z(G)$. Then [8, p. 173] $H_{1} \cap G^{\prime}=1$. Thus there exists $G_{1} \triangleleft G$ such that $G_{1} H=G$ and $G_{1} \cap H_{1}=1$. Let $\chi=\sum_{i=1}^{s} \chi_{i}$, where each $\chi_{i}$ is a irreducible character of $G$. Then for $1 \leqq i \leqq s, \chi_{i \mid H_{1}}=\chi_{i}(1) \mu_{i}$, where $\mu_{i}$ is a linear character of $H_{1}$. For $1 \leqq i \leqq s$, let $v_{i}$ be a character $G / G_{1}$ such that $v_{i \mid H_{1}}=\bar{\mu}_{i}$ and let $\theta_{i}=v_{i} \chi_{i}$. Define $\theta=\sum_{i=1}^{s} \theta_{i}$. Then $\theta$ has $H_{1}$ in its kernel and $\theta_{\mid G_{1}}$ is faithful. Thus if $H_{1} \neq 1$ the theorem may be applied to $G / H_{1}$ which implies that it also 
holds for $G$ contrary to assumption. Hence $H_{1}=1$ as required.

(5C) Let $G_{1} \triangleleft G$ and $G \neq G_{1}$. Let $H_{1}=H \cap G_{1}$. Then there exists a subgroup $L$ of $H_{1}$ such that $L \triangleleft G$ and $\left[H_{1}: L\right]=1$ or pfor some prime $p$.

Proof. Let $L$ be the maximal subgroup of $H_{1}$ which is normal in $G_{1}$. The theorem applied to $G_{1}$ yields that $\left[H_{1}: L\right]=1$ or $p$ for some prime $p$. Thus the Sylow theorems yield that any subgroup of $G_{1}$ whose order divides $h$ is conjugate to a subgroup of $H_{1}$. If $x \in G$ then $x^{-1} L x \subseteq G_{1}$, hence there exists $y \in G_{1}$ such that $y^{-1} x^{-1} L x y \subseteq H_{1}$. Furthermore $y^{-1} x^{-1} L x y \triangleleft G_{1}$. Hence $\left\langle L, y^{-1} x^{-1} L x y\right\rangle \triangleleft G_{1}$. The maximal nature of $L$ now yields that $y^{-1} x^{-1} L x y=L$ and so $x^{-1} L x=y L y^{-1}=L$ as $y \in G_{1}$. As $x$ was an arbitrary element of $G$ we get that $L \triangleleft G$ as required.

(5D) If $G_{1} \triangleleft G$ and $G_{1} \neq G$ then $\left(\left|G_{1}\right|, h\right)=1$ or $p$ for some prime $p$.

Proof. Let $H_{1}=H \cap G_{1}$. If the result is false then $\left|H_{1}\right|$ is not a prime and (5C) yields the existence of a subgroup $L$ of $H$ such that $L \neq 1$ and $L \triangleleft G$. Hence $H \subseteq C_{G}(L) \triangleleft G$. By $(5 \mathrm{~B}) C_{G}(L) \neq G$. Hence (5C) applied to $C_{G}(L)$ yields the existence of a subgroup $L_{1}$ of $H$ such that $L_{1} \triangleleft G$ and $\left[H: L_{1}\right]=1$ or $p$ for some prime $p$. This contradicts the choice of $G$.

(5E) If $L \triangleleft G$ and $|L| \mid h$ then $L=1$.

Proof. Assume that the result is false and let $L \triangleleft G,|L| \mid h, L \neq 1$. Then $L \subseteq H$ and so $H \subseteq C_{G}(L) \triangleleft G$. By (5D) this yields that $C_{G}(L)=G$ contrary to (5B).

(5F) Let $p \mid h$ for some prime $p$ and let $P \neq 1$ be a $p$-group contained in $G$. If $P G_{1}$ is a group, $G_{1} \triangleleft G_{1} P$ and $p \nmid\left|G_{1}\right|$ then $G_{1} \subseteq C_{G}(P)$.

Proof. For any prime $q$ dividing $\left|G_{1}\right|$ there exists a Sylow $q$-group of $G_{1}$ such that $P \subseteq N_{G}(Q)$. Hence $P Q$ is a solvable group and thus [6] $P \triangleleft P Q$. Hence $\left|G_{1}\right||| N_{G_{1} P}(P) \mid$ and so $P G_{1}=P \times G_{1}$.

(5G) $H \subseteq G^{\prime}$.

If the result is false then there exists $G_{1} \triangleleft G$ such that $\left[G: G_{1}\right]=q$, where $q$ is a prime dividing $h$. Let $P_{1}=H \cap G_{1}$. Then by (5D) $\left|P_{1}\right|=p$ for some prime $p$. Now (5F) yields that $p=q$ and $H=P$ is a Sylow $p$-group of $G$. Let $\chi_{1}$ be an irreducible constituent of $\chi$ which does not have $P_{1}$ in its kernel. Let $W$ be the kernel of $\chi_{1}$ and let $\chi=\chi_{1}+\chi_{2}$.

Suppose that $W \neq 1$. Assume first that $(p,|W|)=1$. Then the theorem may be applied to $G / W$ and yields the existence of a subgroup $P_{2}$ of $P, P_{2} \neq 1$ with the property that $P_{2} W / W \triangleleft G / W$. Hence by (5F) $P_{2} W=P_{2} \times W$ and so $P_{2} \triangleleft G$ contrary to assumption. Suppose now that $p|| W \triangleleft \mid$. Let $P_{2}=P \cap W$. If $P_{1} W \triangleleft G$ then by (5D) $P_{1} W=G$. Thus $\left[G: G_{1} \cap W\right]=p^{2}$ and $G_{1} \cap W \triangleleft G$. Hence by (5F) $P \triangleleft G$ contrary to assumption. Thus $P_{1} W / W$ is not normal in $G / W$. Hence Lemma 3.1 yields that $\chi_{1}(1) \geqq(p-1) / 2$. Therefore $\chi_{2}(1)<(p-1) / 2$. Since $\chi_{2 \mid W}$ is a faithful character of $W$ we get [5, Theorem 1] that $P_{2} \triangleleft W$. Hence $P_{2} \triangleleft G$ which is not the case. Consequently $W=1$. Thus the minimal nature of $\chi(1)$ implies that $\chi=\chi_{1}$ is irreducible. 
Since $\chi(1)<p$ we get that $\chi_{\mid G_{1}}$ is irreducible. Hence if

$$
e=\left[N_{G_{1}}\left(P_{1}\right): C_{G_{1}}\left(P_{1}\right)\right]=\left[N_{G}\left(P_{1}\right): C_{G}\left(P_{1}\right)\right]
$$

then by Lemma 3.1

$$
\chi(1)=p-e \text { or } \chi(1)=e=\frac{p-1}{2}
$$

Since $P_{1}$ is not normal in $G$ there exists a prime $q$ such that no Sylow $q$-group of $G_{1}$ normalizes $P_{1}$. If $Q$ is a Sylow $q$-group of $G_{1}$ then $\left[G: N_{G}(Q)\right]=\left[G_{1}: N_{G_{1}}(Q)\right]$. Hence by choosing a suitable conjugate of $Q$ it may be assumed that for some element $x$ of $P-P_{1}, x \in N(Q)$. Hence $\langle x\rangle Q$ is a solvable group thus [6] $\langle x\rangle Q=\langle x\rangle \times Q$. Therefore $\langle P, Q\rangle \subseteq C_{G}(x)$. Thus $P_{1}$, and hence $P$, is not normal in $C_{G}(x)$. Therefore [8, p. 173] $C_{G}(x)=\langle x\rangle \times X$, where $X \subseteq G_{1}$. Hence $P_{1} \subseteq X$ but $P_{1}$ is not normal in $X$. Let $\zeta$ be an irreducible constituent of $\chi_{\mid\langle x\rangle+X}$ which does not contain $P_{1}$ in its kernel. If $V$ is the kernel of $\zeta$ and $P_{1} V \triangleleft X$ then by (5F) $P_{1} \triangleleft X$, which is not the case. Since $N_{G}(P) \subseteq C_{G}(x)$ we have $\left[8\right.$, p. 169] that $e=\left[N_{X}\left(P_{1}\right): C_{X}\left(P_{1}\right)\right]$. Hence by Lemma 3.1 and (5.1) $\chi_{\mid X}$ is irreducible except possibly if $\chi(1)=(p+1) / 2$ and $\zeta(1)=(p-1) / 2$. If $\chi_{\mid X}$ is irreducible then $\chi_{\mid\langle x\rangle \times x}=\lambda \omega$, where $\lambda$ is a linear character of $\langle x\rangle \times X \mid X$ while $\omega$ is an irreducible character of $\langle x\rangle \times X \mid\langle x\rangle$. Hence

$$
|\chi(x)|=|\lambda(x) \omega(1)|=|\chi(1)| .
$$

Therefore $x \in Z(G)$ contrary to (5B).

Assume now that $\chi_{\mid X}$ is reducible. Hence $\chi(1)=(p+1) / 2$. Thus $p>3$ and [7, p. 111] $G_{1} / Z\left(G_{1}\right)$ is isomorphic to $P S L_{2}(p)$ or $A_{6}$ or $A_{7}$ in case $p=5$ or 7 respectively. It is well known that $P S L_{2}(p)$ has no outer automorphism of order $p$ and $A_{6}, A_{7}$ has no outer automorphism of order 5,7 respectively. Hence $G / Z(G)$ is isomorphic to $G_{1} / Z(G) \times P_{2} Z(G) / Z(G)$, where $\left|P_{2}\right|=p$. Thus $P_{2} \triangleleft G$ contrary to (5E). The proof is complete.

$(5 \mathrm{H}) \chi$ is an irreducible character of $G$ and $\chi(1)>3$.

Proof. All 1, 2 and 3 dimensional matrix groups over the complex numbers are known (see [1]). It may be verified by inspection that the theorem holds for these.

Let $\chi_{1}$ be an irreducible constituent of $\chi$, let $\chi=\chi_{1}+\chi_{2}$ and assume that $\chi_{2} \neq 0$. Let $W$ be the kernel of $\chi_{1}$. Since $\chi$ has minimum degree $\chi_{1} \neq 1_{G}$, thus $W \neq G$. Furthermore $W \neq 1$. Thus by $(5 G) W H \neq G$. If $H \cap W=1$, then by (5F) $W H=W \times H$. The theorem applied to $G / W$ yields the existence of a subgroup $L$ such that $L W / W \triangleleft G / W$ and $L=H$ or $[H: L]=p$ for some prime $p$. Thus $L W \triangleleft G$ and $L W=L \times W$. Hence $L \triangleleft G$ contrary to (5E).

Assume now that $K=H \cap W \neq 1$. Then $|K|=p$ for some prime $p$ by (5D). If $K \triangleleft W$, then $K \triangleleft G$ contrary to (5E). Hence [5, Theorem 1] 
$\chi_{2}(1)>(p-1) / 2 \geqq(\chi(1)+1) / 2$. Thus $\chi_{1}(1)<(\chi(1)+1) / 2$. Hence [5, Theorem 1$]$ $H W / W \triangleleft G / W$ and so $H W \triangleleft G$ contrary to (5D). The statement is proved.

At this point it is convenient to consider two cases separately.

Case I. $H$ is not a T.I. set in G.

Case II. $H$ is a T.I. set in $G$.

These cases will be handled in the next two sections. The arguments used to prove the theorem in the two cases are quite similar.

6. The proof of the main theorem: Case I. The notation of $\$ 5$ is used. In addition it is assumed that

(6A) $H$ is not a T.I. set in $G$.

(6B) There exists $z$ in $G$ such that

$$
1 \neq K=H \cap z^{-1} H z \neq H .
$$

Furthermore for any such $z, K$ is a T.I. set in $G . C_{G}(K)=K \times X$ for some nonsolvable group $X$. If $P=H \cap X$, then $|P|=p$ for some prime $p, P$ is not normal in $X$ and $H=K \times P$.

Proof. The existence of such a $z$ follows immediately from (6A). By (5B) $C_{G}(K) \neq G$. Hence the theorem applied to $C_{G}(K)$ yields that $[H: K]=p$ for some prime $p$. Hence $[8$, p. 173$] C_{G}(K)=K \times X$ for some group $X$. Since $\left\langle H, z^{-1} H z\right\rangle \subseteq C_{G}(K)$ we see that $P$ is not normal in $X$ and so [6] $X$ is not solvable. Furthermore $|P|=p$ is a prime and $H=K \times P$. It only remains to show that $K$ is a T.I. set in $G$.

Let $K_{1}=K \cap y^{-1} K y \neq 1$ for some $y \in G$. Then

$$
K_{1}=H \cap z^{-1} H z \cap y^{-1} H y \cap y^{-1} z^{-1} H z y .
$$

By (5B), $C_{G}\left(K_{1}\right) \neq G$, hence the theorem applied to $C_{G}\left(K_{1}\right)$ implies that $\left[H: K_{1}\right]=p$. Thus $K=K_{1}$. Hence $K$ is a T.I. set in $G$.

For the remainder of this section $K$ will denote one of the groups (depending on $z$ ) defined in (6B). Furthermore

$$
\begin{aligned}
N & =N_{G}(K), \\
N_{0} & =\left\{\bigcup_{y \in K^{*}} C_{G}(y)\right\}-Z(G) . \\
\chi_{\mid K \times X} & =\sum_{i=1}^{s} \lambda_{i} \omega_{i},
\end{aligned}
$$

where each $\lambda_{i}$ is a linear character of $K \times X / X$, each $\omega_{i}$ is an irreducible character of $K \times X / K$ and the notation is chosen so that $P$ is not in the kernel of $\omega_{1}$.

(6C) Let $Y$ be the normal subgroup of $X$ generated by $P$. Then $Y$ is in the kernel of each $\omega_{i}$ for $2 \leqq i \leqq s$. Furthermore $\omega_{1 \mid Y}$ is a faithful character of $Y$ and $\omega_{1} \geqq(p-1) / 2$. 
Proof. For $1 \leqq i \leqq s$ let $W_{i}$ be the kernel of $\omega_{i}$. If $\omega_{i}(1)<(p-1) / 2$ then [5, Theorem 1] $P W_{i} \triangleleft X$. Hence if $\omega_{i}(1)<(p-1) / 2$ for $1 \leqq i \leqq s$ then $P=\bigcap_{i=1}^{s} P W_{i} \triangleleft X$ contrary to (6B). Since $\chi(1)<p-1$, this implies that there exists exactly one $k$ with $1 \leqq k \leqq s$ such that $\omega_{k}(1) \geqq(p-1) / 2$. Furthermore $P W_{k}$ is not normal in $X$. Let $W=\bigcap_{i \neq k} W_{i}$. Then $P W \cap W_{k}=1$. If $P \notin W$ then (5F) implies that $P \triangleleft P W$. Thus $P \triangleleft X$ contrary to (6B). Thus $P \subseteq W$ and so $Y \subseteq W$. Hence $k=1$ and the statement is proved.

(6D) If $x \in K$ then $x$ is not conjugate to any element of $H-K$. In particular $N_{G}(H) \subseteq N$.

Proof. Suppose first that $|K| \neq p$. If $z^{-1} x z \in H$ for some $z \in G$, then [8, p. 169] it may be assumed that $z \in N_{G}(H)$. Hence $z^{-1} K z \subseteq H$. As $[H: K]=\left[H: z^{-1} K z\right]=p$ and $|K| \neq p$, this yields that $K \cap z^{-1} K z \neq 1$. Thus by (6B) $z \in N$ and $z^{-1} x z \in K$ as required.

Assume now that $|K|=p$ and for some $k$

$$
z^{-1} x z=x^{k} y, \quad y \in P^{*} \text {. }
$$

By (6.1) and (6C)

$$
\begin{gathered}
\chi\left(x^{j}\right)=\omega_{1}(1) \lambda_{1}\left(x^{j}\right)+\sum_{i=2}^{s} \omega_{i}(1) \lambda_{i}\left(x^{j}\right), \\
\chi\left(\left(x^{k} y\right)^{j}\right)=\omega_{1}\left(y^{j}\right) \lambda_{1}\left(x^{k j}\right)+\sum_{i=2}^{s} \omega_{i}(1) \lambda_{i}\left(x^{k j}\right)
\end{gathered}
$$

for $0 \leqq j \leqq p-1$. Hence (6C) and (6.3) imply that $\chi_{\mid\langle x\rangle}$ contains some linear character with multiplicity at least $\omega_{1}(1) \geqq(p-1) / 20$ However Lemma 3.1 implies that $\omega_{1} \lambda_{1 \mid\left\langle x^{k} y\right\rangle}$ is a sum of $\omega_{1}(1)$ distinct linear characters of $\left\langle x^{k} y\right\rangle$. As $\chi(1)<p-1,(6.2)$ and (6.4) now yield that $\chi(1)=p-2, \omega_{1}(1)=(p-1) / 2$ and either $\lambda_{i \mid\langle x\rangle}=\lambda_{2 \mid\langle x\rangle}$ for $2 \leqq i \leqq s$ or $x^{k}=1$. If $x^{k} \neq 1$ then by $(6.3), \chi_{\mid\langle x\rangle}$ has at most two distinct irreducible constituents. By (6.2) this must then also be the case for $\chi_{\mid\left\langle x^{k} y\right\rangle}$. Hence Lemma 3.1 and (6.4) yield that $\omega_{1}(1) \leqq 2$. Thus $p \leqq 5$ and so $\chi(1) \leqq 3$ contrary to $(5 \mathrm{H})$.

Assume now that $x^{k}=1$. Then since $\omega_{1}(1)=(p-1) / 2$ and $\chi(1)=p-2$ it follows from Lemma 3.1 and (6.4) that $\left(\chi_{\mid\langle y\rangle}, 1_{\langle y\rangle}\right)=(p-3) / 2$. Hence by (6.2) and (6.3) $\lambda_{1 \mid\langle x\rangle} \neq 1_{\langle x\rangle}$ and $\lambda_{i \mid\langle x\rangle}=1_{\langle x\rangle}$ for $2 \leqq i \leqq s$. Thus by $(6.4) \chi_{\mid\langle y\rangle}$ contains no nonprincipal irreducible character with multiplicity greater than 1 , while by (6.3) $\chi_{\mid\langle x\rangle}$ contains $\lambda_{1 \mid\langle x\rangle}$ with multiplicity $\omega_{1}(1)$. This contradicts (6.2) and suffices to prove (6D).

(6E) $N_{0} \subseteq N_{G}\left(N_{0}\right)=N$.

Proof. Clearly $N \subseteq N_{G}\left(N_{0}\right)$. Since $K$ is a T.I. set $N_{0} \subseteq N$. It remains to show that $N_{G}\left(N_{0}\right) \subseteq N$. Let $y \in N_{G}\left(N_{0}\right)$. If $x \in K$ then the order of $y^{-1} x y$ divides $h$. As $y^{-1} x y \in N_{0} \subseteq N$ this implies that $y^{-1} x y \in C_{G}(K)$. Hence there exists $z \in C_{G}(K)$ such that $z^{-1} y^{-1} x y z \in H$. Now (6D) implies that $z^{-1} y^{-1} x y z \in K$ and so 
$y^{-1} x y \in z K z^{-1}=K$. Thus $y \in N$. Since $y$ was chosen arbitrarily in $N_{G}\left(N_{0}\right)$ the result follows.

(6F) $\lambda_{1}=1_{K \times X}$.

Proof. Let $\chi_{\mid N}=\Sigma_{i} \zeta_{i}$, where each $\zeta_{i}$ is an irreducible character of $N$ and $\zeta_{1 \mid K \times X}$ contains $\lambda_{1} \omega_{1}$ as a constituent. By (6C) $\zeta_{1 \mid K \times X}=\lambda_{1} \omega_{1}$ since $K \times X \triangleleft N$. Let $K_{1}$ be the kernel of $\lambda_{1 \mid K}$, then $K \cap N^{\prime} \subseteq K_{1}$. By (5G) $K \subseteq G^{\prime}$. Hence [8, p. 173], $K \subseteq N_{G}(H)^{\prime}$. Thus by (6D) $K \subseteq N^{\prime}$ and so $K=K_{1}$. This proves the statement.

(6G) If $x \in N_{0}$ then $C_{G}(x) \subseteq N$.

Proof. Suppose $x \in N_{0}$ such that $C_{G}(x) \neq N$. Let $K_{0}=K \cap C_{G}(x)$. By definition of $N_{0}, C_{G}(x) \neq G$. Hence if $\left|K_{0}\right|$ is not a prime the theorem applied to $C_{G}(x)$ yields the existence of a subgroup $K_{1}$, such that $1 \neq K_{1} \subseteq K_{0}$ and $K_{1} \triangleleft C_{G}(x)$. Since $K$ is a T.I. set in $G$ this implies that $C_{G}(x) \subseteq N_{G}\left(K_{1}\right) \subseteq N$ contrary to assumption. Therefore $\left|K_{0}\right|=q$ is a prime.

Assume first that $K_{0}=C_{G}(x) \cap H$. Let $\zeta$ be an irreducible constituent of $\chi_{\mid C_{G}(x)}$ which does not have $K_{0}$ in its kernel. Let $W$ be the kernel of $\zeta$. Then since $K_{0}$ is not normal in $C_{G}(x),(5 F)$ implies that $K_{0} W / W$ is not normal in $C_{G}(x) / W$. Hence Lemma 3.1 applied to $C_{G}(x) / W$ implies that $\zeta_{\mid K_{0}}$ contains at least $(q-1) / 2 \geqq(\chi(1)+1) / 2$ nonprincipal characters of $K_{0}$ as constituents. However (6C) and(6F) imply that $\chi_{\mid K_{0}}$ contains $1_{K_{0}}$ with multiplicity at least $(p-1) / 2 \geqq(\chi(1)+1) / 2$. Hence $\chi_{\mid K_{0}}(1) \geqq \chi(1)+1$ which is nonsense. Thus $K_{0} \neq C_{G}(x) \cap H$.

Let $H_{0}=C_{G}(x) \cap H$. Then $\left[H_{0}: K_{0}\right]=p$. Suppose now that $K \neq K_{0}$. The theorem applied to $C_{G}(x)$ yields the existence of a subgroup $H_{1}$ such that $1 \neq H_{1} \subseteq H$ and $H_{1} \triangleleft C_{G}(x)$. Therefore $\left\langle H, C_{G}(x)\right\rangle \subseteq N_{G}\left(H_{1}\right)$. By $(5 E) N_{G}\left(H_{1}\right) \neq G$ and the theorem applied to $N_{G}\left(H_{1}\right)$ yields the existence of a subgroup $\mathrm{H}_{2}$ of $\mathrm{H}$ such that $\left[H: H_{2}\right]$ is a prime or $H=H_{2}$ and $H_{2} \triangleleft N_{G}\left(H_{1}\right)$. Since $|K|$ is not a prime $K_{2}=K \cap H_{2} \neq 1$. As $K$ is a T.I. set in $G$, and therefore also in $N_{G}\left(H_{1}\right)$, this implies that $K \subseteq H_{2}$. Hence $H_{2}=K$ or $H_{2}=H$. As $C_{G}(x) \subseteq N_{G}\left(H_{1}\right) \subseteq N_{G}\left(H_{2}\right)$ it now follows from (6D) that in either case $C_{G}(x) \subseteq N$ contrary to the choice of $x$. Thus $K=K_{0}$ and $H=H_{0}$.

Since $H \triangleleft C_{G}(x)$ there exists $H_{1}$ such that $1 \neq H_{1} \subset H$ and $H_{1} \triangleleft C_{G}(x)$. Furthermore $H_{1} \neq K$. Now $H_{1}$ can play the role of $K$ in (6B). Thus (6.1), (6C) and (6F) applied to $H_{1}$ yield that

$$
\left(\chi_{\mid H_{1}}, 1_{H_{1}}\right) \geqq \frac{\chi(1)+1}{2}
$$

Let $H_{1}=\langle x y\rangle$, where $x \in K$ and $y \in P^{\#}$. Then (6.1), (6C) and (6F) yield that

$$
\chi(x y)=\omega_{1}(y)+\sum_{i=2}^{s} \omega_{i}(1) \lambda_{i}(x) .
$$

Since $y \neq 1$, Lemma 3.1 and (6.6) imply that $\chi_{\mid H_{1}}$ contains at least 
$\omega_{1}(1) \geqq(\chi(1)+1) / 2$ nonprincipal linear characters of $H_{1}$ as constituents. Thus (6.5) implies that $\chi_{\mid H_{1}}(1) \geqq \chi(1)+1$. The proof of $(6 \mathrm{G})$ is complete in all cases. (6H) $N_{0}$ is a T.I. set in $G$.

Proof. By (6E) $N_{0} \subseteq N_{G}\left(N_{0}\right)$. Suppose that $1 \neq x \in N_{0} \cap y^{-1} N_{0} y$. Then $y x y^{-1} \in N_{0}$. Let $K_{1}=K \cap C_{G}\left(y x y^{-1}\right)$. Thus $y^{-1} K_{1} y \subseteq C_{G}(x)$. Hence by $(6 \mathrm{G})$ $y^{-1} K_{1} y \subseteq N$. As $\left|y^{-1} K_{1} y\right| \mid h$ we get that $y^{-1} K_{1} y \subseteq C_{G}(K)$. Thus there exists $z \in C_{G}(K)$ such that $z^{-1} y^{-1} K_{1} y z \subseteq H$. Hence by (6D) $z^{-1} y^{-1} K_{1} y z \subseteq K$. Therefore $y^{-1} K_{1} y \subseteq z K z=K$. As $K$ is a T. I. set in $G$ this implies that $y \in N$. Hence by (6E) $y \in N_{G}\left(N_{0}\right)$.

The proof of the theorem in case I can now be completed. By (6B), (6E) and $(6 \mathrm{H})$ Hypothesis 4.1 (i) and (ii) are satisfied where $K$ plays the role of $Y$. By assumption, Hypothesis 4.1 (iii) is satisfied.

By $(5 \mathrm{H}) \chi$ is irreducible.

Hence by Lemma 4.1

$$
\left(\chi_{\mid K}, 1_{K}\right)<\frac{\chi(1)}{|K|^{1 / 2}}<\frac{p-1}{|K|^{1 / 2}} .
$$

However (6C) and $(6 \mathrm{~F})$ yield that

$$
\left(\chi_{\mid K}, 1_{K}\right) \geqq \frac{p-1}{2} .
$$

Consequently $\left((p-1) / 2<(p-1) /|K|^{1 / 2}\right.$, or $|K|<4$. Hence $\chi(1) \leqq|K|-1<3$ contrary to $(5 \mathrm{H})$. The theorem is proved in case $\mathrm{I}$.

7. The Proof of the main theorem: Case II. The notation of $\S 5$ is used. In addition it is assumed that

(7A) $H$ is a T.I. set in $G$.

Define

$$
\begin{aligned}
& N=N_{G}(H) \\
& N_{0}=\left\{\bigcup_{y \in H^{*}} C_{G}(y)\right\}-Z(G)
\end{aligned}
$$

(7B) $N_{0} \subseteq N=N_{G}\left(N_{0}\right)$.

Proof. Clearly $N \subseteq N_{G}\left(N_{0}\right)$. Since $H$ is a T. I. set in $G, N_{0} \subseteq N$. If $x \in N_{G}\left(N_{0}\right)$ and $y \in H$, then $x^{-1} y x$ has order dividing $h$ and $x^{-1} y x \in N_{0}$. As $N_{0} \subseteq N$ every element of $N_{0}$ whose order divides $h$ is in $H$. Thus $x^{-1} y x \in H$. Hence $x \in N$. Therefore $N_{G}\left(N_{0}\right) \subseteq N$ as required.

(7C) $N_{0}$ is not a T.I. set in $G$.

Proof. If $N_{0}$ is a T.I. set in $G$ then by (7B) Hypothesis 4.1 is satisfied. Since $|H|$ is not a prime (5D) implies that $G$ is the normal subgroup generated by $H$. Hence by Lemma $4.2 \chi(1)+1>|H|^{1 / 2}$. Thus if $|H|$ is divisible by exactly $t$ 
primes then $\chi(1)+1>\{\chi(1)+1\}^{t / 2}$. Hence $t<2$ and $|H|$ is a prime contrary to assumption.

(7D) There exists $x \in N_{0}$ such that $C_{G}(x) \ddagger N$.

Proof. Suppose that $C_{G}(x) \subseteq N$ for every $x \in N_{0}$. By (7B) and (7C) there exists $y \notin N$ and $1 \neq x \in N_{0} \cap y^{-1} N_{0} y$. Let $H_{1}=H \cap C_{G}\left(y x y^{-1}\right)$. Thus $y^{-1} H_{1} y \subseteq C_{G}(x) \subseteq N$. As $\left|y^{-1} H_{1} y\right| \mid h$ we see that $y^{-1} H_{1} y \subseteq H$. Thus $y \in N=N_{G}(H)$ since $H$ is a T. I. set in $G$. This contradicts the choice of $y$.

(7E) If $x \in N_{0}$ and $C_{G}(x) \nsubseteq N$ then $C_{G}(x) \cap H$ has prime order. Furthermore $C_{G}(x) \cap H$ is not normal in $C_{G}(x)$.

Proof. Let $H_{1}=H \cap C_{G}(x)$. Thus $H_{1} \neq 1$. If $\left|H_{1}\right|$ is not a prime then the theorem applied to $C_{G}(x)$ yields the existence of a subgroup $\mathrm{H}_{2}$ such that $1 \neq H_{2} \subseteq H_{1}$ and $H_{2} \triangleleft C_{G}(x)$. Hence $C_{G}(x) \subseteq N_{G}\left(H_{2}\right)$. Thus $C_{G}(x) \subseteq N$ as $H$ is a T.I. set in $G$, contrary to assumption.

(7F) Suppose that $x \in N_{0}$ and $C_{G}(x) \nsubseteq N$. Let $C_{G}(x) \cap H=P$ and $|P|=p$. Then $H=K \times P$ for some group $K$ and $N_{G}(P)=N_{G}(K)=N$. Furthermore

$$
\left(\chi_{\mid P}, 1_{P}\right)_{P}<\frac{\chi(1)+1}{2} \leqq \frac{p-1}{2},
$$

and

$$
\left(\chi_{\mid K}, 1_{K}\right)_{K} \geqq \frac{p-1}{2}
$$

Proof. Let $C=C_{G}(x)$. By (7E) $|P|=p$ is a prime. Let

$$
\chi_{\mid c}=\omega+\mu,
$$

where $\mu$ is a character of $C$ which has $P$ in its kernel and $\omega$ is a sum of irreducible characters none of which have $P$ in their kernel. Let $\omega_{1}$ be an irreducible constituent of $\omega$ and let $W$ be the kernel of $\omega_{1}$. If $W P \triangleleft C$, then by (5F) $P \triangleleft C$ contrary to assumption. Hence Lemma 3.1 applied to $C / W$ implies that $\omega_{1}(1) \geqq(p-1) / 2$. As $\omega(1)<p-1$ this yields that $\omega=\omega_{1}$ is an irreducible character of $C$. Since $\chi$ is a class function of $G$, Lemma 3.1 implies that

$$
e=\left[N_{G}(P): C_{G}(P)\right]=\left[N_{C}(P): C_{C}(P)\right] .
$$

By (7.4) there exists a cyclic subgroup $E_{1}$ of $C$ such that $E_{1} \subseteq N_{G}(P)$ and

$$
e=\left[N_{G}(P) \cap E_{1} P: C_{G}(P) \cap E_{1} P\right] \text {. }
$$

Since $P=Z(H\langle x\rangle)$ we have $[8$, p. 173] that $H=P \times K$ for some group $K$, $K \triangleleft H\langle x\rangle$ and $K$ is uniquely determined by these conditions. Furthermore there exists a subgroup $H_{1}$ of $H$ such that $H=P \times H_{1}$ and $H_{1} \triangleleft N_{G}(P)$. Hence $H_{1} \triangleleft H\langle x\rangle$, thus the uniqueness of $K$ implies that $K=H_{1}$. Hence $K \triangleleft N_{G}(P)$. Using the same argument we get the existence of $P_{1}$ in $H$ such that $K \times P_{1}=H$ 
and $P_{1} \triangleleft N_{G}(K)$. Therefore $P=P_{1}$ since $P=Z(H\langle x\rangle)$. Consequently since $H$ is a T.I. set in $G$,

$$
N_{G}(P)=N_{G}(K) \subseteq N
$$

Furthermore (7E) implies that

$$
C_{G}\left(x^{k}\right) \cap K=1 \text { for } x^{k} \notin Z(G) .
$$

Define

$$
X=\langle x\rangle K P E_{1} .
$$

Let

$$
\chi_{\mid X}=\alpha+\beta,
$$

where $\beta$ is a character which has $P$ in its kernel and $\alpha$ is a sum of irreducible characters none of which have $P$ in their kernel. Comparing (7.3) and (7.7) we get that

$$
\chi_{\mid\langle x\rangle P E_{1}}=\omega_{\mid\langle x\rangle P E_{1}}+\mu_{\mid\langle x\rangle P E_{1}}=\alpha_{\mid\langle x\rangle P E_{1}}+\beta_{\mid\langle x\rangle P E_{1}} .
$$

Hence by Lemma 3.1

or

$$
\omega_{\mid P}=\alpha_{\mid P}, \quad \omega(1)=e=\frac{p-1}{2}
$$

$$
\omega_{\mid P}=\alpha_{\mid P}+1_{P}, \quad \omega(1)=p-e \geqq \frac{p+1}{2} .
$$

Therefore (7.8) implies that

$$
\omega_{\mid\langle x\rangle P E_{1}}=\alpha_{\mid\langle x\rangle P E_{1}}, \quad \omega(1)=e=\frac{p-1}{2}
$$

or

$$
\omega_{\mid\langle x\rangle P E_{1}}=\alpha_{\mid\langle x\rangle P E_{1}}+\lambda, \quad \omega(1)=p-e \geqq \frac{p+1}{2},
$$

where $\lambda$ is a linear character of $\langle x\rangle P E_{1}$ which has $P$ in its kernel. Therefore in any case Lemma 3.1 implies that

$$
\left(\alpha_{\mid P}, 1_{P}\right)=0, \quad \alpha(1) \geqq \frac{p-1}{2} .
$$

Since $x \in Z(C)$ and since $\omega$ is an irreducible character of $C$, it follows from (7.9) and (7.10) that $|\alpha(x)|=\alpha(1)$. Thus if $W$ is the kernel of $\alpha_{\mid\langle x\rangle K}$, then $x W / W \in Z(\langle x\rangle K / W)$. Therefore by (7.6)

$$
\alpha_{\mid K}=\alpha(1) 1_{K}
$$


Now (7.1) follows from (7.11) since $\chi(1)<p-1$, while (7.2) follows from (7.11) and (7.12).

In view of (7.5) it only remains to show that $K \triangleleft N$. Let $y \in K$ and let $z \in N$. Then $z^{-1} y z \in H$. Assume that $z^{-1} y z \notin K$. By definition $\left(\alpha_{\mid H}, 1_{H}\right)=0$. Thus by (7.12) $\left(\alpha_{\mid H_{1}}, 1_{H_{1}}\right)=0$ for any subgroup $H_{1}$ of $H$ which is not contained in $K$. Hence $\left(\alpha_{\mid\langle z-1}-1_{y z\rangle}, 1_{\langle z-1 y z\rangle}\right)=0$. Hence (7.7) and (7.11) imply that

$$
\left(\chi_{\mid\langle z-1}, 1_{\langle z-1}, 1_{y z\rangle}\right)<\frac{p-1}{2} .
$$

Therefore $\left(\chi_{\mid\langle y\rangle}, 1_{\langle y\rangle}\right)<(p-1) / 2$ contrary to (7.2). Thus $z^{-1} y z \in K$. Hence $z \in N_{G}(K)$ and $K \triangleleft N$ as required. The statement is proved.

Let $K$ be a fixed group defined by $(7 \mathrm{~F})$. Define

$$
N_{1}=\left\{\bigcup_{y \in K^{*}} C_{G}(y)\right\}-Z(G) .
$$

(7G) If $x \in N_{1}$ then $C_{G}(x) \subseteq N$.

Proof. Suppose that $x \in N_{1}$ and $C_{G}(x) \nsubseteq N$. Let $Q=C_{G}(x) \cap K$. By (7E) $|Q|=q$ is a prime and $Q=C_{G}(x) \cap H$. Hence (7.1) applied to $Q$ in place of $P$ yields that $\left(\chi_{\mid Q}, 1_{Q}\right)<(\chi(1)+1) / 2$. However since $Q \subseteq K$, (7.2) implies that $\left(\chi_{\mid Q}, 1_{Q}\right) \geqq(\chi(1)+1) / 2$. This contradicts the assumption and establishes the result.

(7H) $K$ is a T.I. set in $G$ and $N_{1} \subseteq N_{G}\left(N_{1}\right)=N_{G}(K)=N$.

Proof. Clearly $K \subseteq N_{G}(K)$. If $1 \neq x \in K \cap z^{-1} K z$, then $z \in N$. Hence by (7F) $z \in N_{G}(K)$ and $K$ is a T.I. set in $G$.

Clearly $N \subseteq N_{G}\left(N_{1}\right)$. Since $K$ is a T. I. set $N_{1} \subseteq N$. It remains to show that $N_{G}\left(N_{1}\right) \subseteq N$. Let $y \in N_{G}\left(N_{1}\right)$ and let $z \in K$. Then the order of $y^{-1} z y$ divides $h$ and $y^{-1} z y \in N_{1}$. Thus $y^{-1} z y \in H$. Hence, $y \in N$ since $H$ is a T.I. set in $G$.

(7I) $N_{1}$ is a T.I. set in $G$.

Proof. By (7H) $N_{1} \subseteq N_{G}\left(N_{1}\right)$. Suppose that $1 \neq x \in N_{1} \cap y^{-1} N_{1} y$. Then $y x y^{-1} \in N_{1}$. Let $K_{1}=K \cap C_{G}\left(y x y^{-1}\right)$. Thus by (7G) $y^{-1} K_{1} y \subseteq C_{G}(x) \subseteq N$. As $\left|y^{-1} K_{1} y\right| \mid h$, this yields that $y^{-1} K_{1} y \subseteq H$. As $H$ is T.I. set in $G$ we get that $y \in N=N_{G}\left(N_{1}\right)$ by $(7 \mathrm{H})$.

The proof of the theorem in Case II can now be completed. By (7H) and (7I) Hypothesis 4.1 is satisfied with $K$ and $N_{1}$ in the role of $Y$ and $N_{0}$ respectively. By $(5 \mathrm{H}) \chi$ is irreducible.

Thus by Lemma 4.1!

$$
\left(\chi_{\mid K}, 1_{K}\right)<\frac{1}{|K|^{1 / 2}} \chi(1)<\frac{p-1}{|K|^{1 / 2}} .
$$

Hence (7.2) implies that $(p-1) / 2<(p-1) /|K|^{1 / 2}$. Thus $|K|<4$ and so $\chi(1)<|K|-1<3$ contrary to $(5 \mathrm{H})$. This completes the proof of the theorem in Case II.

The results of $\S \S 6$ and 7 suffice to prove the theorem in all cases. 


\section{REFERENCES}

1. H. F. Blichfeldt, Finite collineation groups, Univ. of Chicago, Chicago, Ill., 1917.

2. R. Brauer, On groups whose orders contain a prime number to the first power. I, Amer. J. Math. 64(1942), 401-420.

3. - On groups whose orders contain a prime number to the first power. II, Amer. J. Math. 64(1942), 421-440.

4. W. Burnside, Theory of groups of finite order, Dover, New York, 1955.

5. W. Feit and J. G. Thompson, Groups which have a faithful representation of degree less than (p-1)/2, Pacific J. Math. 11(1961), 1257-1262.

6. N. Ito, On a theorem of H. F. Blichfeldt, Nagoya Math. J. 5(1954), 75-77.

7. H. F. Tuan, On groups whose order contains a prime number to the first power, Ann. of Math. (2) 45(1944), 110-140.

8. H. Zassenhaus, The theory of groups, Chelsea, New York, 1958.

\section{HARVARD University,}

Cambridge, MassachusetTs 